INFLAMMATORY BOWEL DISEASE

\title{
Anti-Saccharomyces cerevisiae antibodies in twins with inflammatory bowel disease
}

\author{
J Halfvarson, A Standaert-Vitse, G Järnerot, B Sendid, T Jouault, L Bodin, A Duhamel, \\ J F Colombel, C Tysk, D Poulain
} See end of article for
authors' affiliations

Correspondence to: Dr J Halfvarson, Division of Gastroenterology, Department of Internal Medicine, Örebro University Hospital, Örebro 70185, Sweden; jonas.halfvarson@ orebroll.se

Revised version received 20 April 2005 Accepted for publication 21 April 2005 Published online first 29 April 2005
Background and aims: An increased occurrence of anti-Saccharomyces cerevisiae antibodies (ASCA) is reported in unaffected members of families with Crohn's disease. Whether ASCA is a familial trait due to genetic factors or is caused by exposure to environmental factors is unknown. To assess the genetic influence of ASCA we studied its occurrence in a twin population.

Patients and methods: ASCA were analysed in 98 twin pairs with inflammatory bowel disease and were related to clinical phenotype and CARD15/NOD2 genotype.

Results: ASCA were more common in Crohn's disease than in ulcerative colitis (40/70 (57\%) twins v 5/43 (12\%) twins). Associations with ileal Crohn's disease, stricturing/penetrating behaviour, and young age, but not CARD15/NOD2 were confirmed. ASCA were found in 1/20 (5\%) healthy siblings in discordant monozygotic pairs with Crohn's disease compared with 7/27 (26\%) in discordant dizygotic pairs. Using the intraclass correlation coefficient (ICC), no agreement in ASCA titres was observed in discordant twin pairs with Crohn's disease, in monozygotic (ICC $=-0.02)$ or dizygotic (ICC $=-0.26)$ pairs. In contrast, strong agreement was seen within concordant monozygotic twin pairs with Crohn's disease (ICC $=0.76)$. Conclusions: These findings question the concept of ASCA as a marker of genetic susceptibility for Crohn's disease. The agreement in ASCA titres within concordant monozygotic twin pairs with Crohn's disease, suggests that the level of increase is genetically determined. We propose that ASCA are a marker of a response to an environmental antigen and that a specific gene(s) other than CARD15/NOD2 determines the level of response and perhaps also specific phenotypic characteristics.

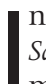
recent years it has been firmly established that antiSaccharomyces cerevisiae antibodies (ASCA) are a serological marker of Crohn's disease (CD). An association between CD clinical phenotypes and ASCA has also been reported. ASCA have qualitatively and quantitatively been associated with young age at onset, ${ }^{12}$ ileal disease, ${ }^{13}{ }^{3}$ and stricturing as well as penetrating disease behaviour. ${ }^{2-4}$

The strongest risk factor for inflammatory bowel disease (IBD) is having a relative with the disease with a relative risk in siblings of $25-42$ for $\mathrm{CD}$ and $8-15$ for ulcerative colitis (UC) compared with the general population. ${ }^{5}$ In recent years, there has been great interest in searching for subclinical markers of IBD in families. Their presence in unaffected members may either indicate genetic and/or environmental factors predisposing to a disease, or identify those in whom an early asymptomatic phase of the disease process is occurring. In a first set of French CD families, ASCA were detected in $69 \%$ of patients with CD and in $20 \%$ of healthy relatives. ${ }^{6}$ The presence of ASCA in healthy relatives was observed in 12 of 20 families and was not restricted to a few particular multiplex families. These findings were confirmed by Seibold and colleagues ${ }^{7}$ who found ASCA in 25\% of 193 healthy first degree relatives. In the study by Sutton and colleagues, ${ }^{8}$ significant familial aggregation of ASCA levels was observed for affected relatives and was even stronger for unaffected relatives. Familial aspects of ASCA were further investigated in a large series of Belgian families having one, two, or more than two affected members. Overall, ASCA prevalence was the same in both sporadic (63.4\%) and familial $(62.1 \%)$ CD. ${ }^{9}$ Within pure CD families, ASCA were present in $54.2 \%$ of $C D$ patients with two members affected versus $74.7 \%$ in $\mathrm{CD}$ patients with three or more members affected. These data further support the suggestion that
ASCA reflects the familial load of the disease. Whether ASCA is a familial trait due to a genetic factor or to increased exposure to an environmental factor is unknown. Twin studies could be of value in this respect. Monozygotic twins have identical genes and share environmental factors while dizygotic twins share environment but only half of the genes are common.

The aim of this study was to evaluate the genetic influence on the occurrence of ASCA in a twin population.

\section{METHODS}

\section{Twins}

Twins were derived from two Swedish cohorts of twins with IBD, both described previously. ${ }^{10-12}$ In brief, twin pairs where at least one twin in each pair had been hospitalised for IBD were identified by running the Swedish twin registry against the Swedish Hospital Discharge Register. The first cohort ( $\mathrm{n}=80$ twin pairs) was identified in 1984 and the second cohort ( $\mathrm{n}=124$ twin pairs) in 2000. A questionnaire was sent to all twins, including questions on diagnosis of IBD and general gastrointestinal symptoms. At the same time, consent from each twin to read his/her medical notes was requested. After responding to the questionnaire and obtaining written consent, the medical notes from twins with IBD or any history of gastrointestinal symptoms were scrutinised to verify or refute the diagnosis of IBD and to characterise the disease phenotypically. For CD, the Vienna classification was used..$^{13}$ Concordant pairs refer to twin pairs where both twins are affected and in discordant pairs only one twin is affected.

Abbreviations: ASCA, anti-Saccharomyces cerevisiae antibodies; $C D$, Crohn's disease; IBD, inflammatory bowel disease; UC, ulcerative colitis; ICC, intraclass correlation coefficient 


\begin{tabular}{|c|c|c|c|}
\hline & $\begin{array}{l}\text { Twin individuals } \\
(\mathrm{n}=70)^{*}\end{array}$ & $\begin{array}{l}\text { ASCA }+ \\
(\mathrm{n}=40)\end{array}$ & $\begin{array}{l}\text { ASCA- } \\
(n=30)\end{array}$ \\
\hline \multicolumn{4}{|l|}{ Age at diagnosis } \\
\hline$<40$ y $(\mathrm{A} 1)$ & 55 (79\%) & $35(50 \%)$ & $20(29 \%)$ \\
\hline$\geqslant 40$ y (A2) & $15(21 \%)$ & $5(7 \%)$ & $10(14 \%)$ \\
\hline \multicolumn{4}{|l|}{ Location } \\
\hline Terminal ileum (LI) & $28(40 \%)$ & $20(29 \%)$ & $8(11 \%)$ \\
\hline Colon (L2) & $21(30 \%)$ & $6(9 \%)$ & $15(21 \%)$ \\
\hline Ileocolon (L3) & $19(27 \%)$ & $12(17 \%)$ & $7(10 \%)$ \\
\hline Upper GI (L4) & $2(3 \%)$ & $2(3 \%)$ & $0(0 \%)$ \\
\hline \multicolumn{4}{|l|}{ Behaviour } \\
\hline Non-stricturing non-penetrating (B1) & $30(43 \%)$ & $12(17 \%)$ & $18(26 \%)$ \\
\hline Stricturing (B2) & $21(30 \%)$ & $16(23 \%)$ & $5(7 \%)$ \\
\hline Penetrating (B3) & $19(27 \%)$ & $12(17 \%)$ & $7(10 \%)$ \\
\hline \multicolumn{4}{|l|}{ CARD15/NOD2 genotype $\dagger$} \\
\hline Wild-type & $56(82 \%)$ & $34(50 \%)$ & $22(32 \%)$ \\
\hline Heterozygote & $12(18 \%)$ & $6(9 \%)$ & $6(9 \%)$ \\
\hline Homozygote & $0(0 \%)$ & $0(0 \%)$ & $0(0 \%)$ \\
\hline $\begin{array}{l}\text { Percentages refer to total number of twi } \\
\text { "Data on CARD15/NOD2 genotype bo } \\
\text { †Arg702Trp, Gly908Arg, or Leu1007f }\end{array}$ & dividuals only. & & \\
\hline
\end{tabular}

Zygosity classification was based on the method applied by the Swedish twin registry. It relies on questions on childhood resemblance and has been shown to be very accurate. ${ }^{14}{ }^{15} \mathrm{~A}$ total of 151 twin pairs of the same sex, born between 1920 and 1980, with known zygosity, had approved further contact and were invited to take part in a study on ASCA.

\section{ASCA}

ASCA detection was performed as previously described. ${ }^{6}{ }^{16}$ Briefly, antigens consisted of phosphopeptidomannan extracted from yeast cells of the $S$ cerevisiae Sul strain from cultures grown in bioreactors. Microtitration plates were coated with $100 \mu \mathrm{l}$ of phosphopeptidomannan at a concentration of $1 \mu \mathrm{g} / \mathrm{ml}$ in sodium carbonate buffer $(60 \mathrm{mmol} / \mathrm{l}$, $\mathrm{pH}$ 9.6) for one hour at $37^{\circ} \mathrm{C}$ and overnight at $4^{\circ} \mathrm{C}$, in moist chambers, and then washed four times in $50 \mathrm{mmol} / \mathrm{l}$ Tris HCL, $150 \mathrm{mmol} / \mathrm{L} \mathrm{NaCl}$, and 0.05\% Tween 20 (TNT) (pH 7.5). Patients' sera were diluted 1:101 in TNT and tested. After washing, $100 \mu \mathrm{l}$ per well of a ready to use horseradish peroxidase labelled goat antihuman immunoglobulin (IgG, IgA, IgM; $\mathrm{H}$ and L chains; Zymed, Biosoft, Paris, France) were added, thereby detecting whole Ig ASCA, including both IgG and IgA. A colour reaction was obtained by using substrate chromogen (tetramethylbenzidine $+\mathrm{H}_{2} \mathrm{O}_{2}$ ) for horseradish peroxidase. Optical density was read at $\lambda=450 / 620 \mathrm{~nm}$. Internal titred standards were used for standardising the test. Results of individual sera were expressed in U/ml extrapolated from the standard curve. The cut off was set at $7.2 \mathrm{U} / \mathrm{ml}$.

\section{CARD 15/NOD2 polymorphisms}

Data on CARD15/NOD2 status was available from two previous studies. Genotyping was performed by polymerase chain reaction-restriction fragment length polymorphism in monozygotic twins ${ }^{17}$ and by polymerase chain reactionsequence specific primers in dizygotic twins. ${ }^{18}$

\section{Statistics}

Differences in the presence of ASCA and other categorical data were analysed by Fisher's exact test, adopted for small samples and for tables larger than $2 \times 2$, using StatXact version 6 (Cytel Software Corporation, Cambridge, Massachusetts, USA). Levels of ASCA titres were also evaluated in a quantitative multivariate perspective. Mean ASCA titres with corresponding 95\% confidence interval (CI) for the different twin groups and estimates of the differences between the groups were analysed using regression analysis of a mixed model design with allowance for dependence within the twin pair, as well as with or without additional explanatory variables apart from those of diagnosis. ${ }^{19}$ Due to the complexity of the model and the limited number of twins, all clinical characteristics could not be included as explanatory variables. Location of CD (two categories-colonic disease (L2) and small bowel involvement (L1+L3+L4)) and behaviour (two categories-non-stricturing non-penetrating (B1) and stricturing or penetrating behaviour $(\mathrm{B} 2+\mathrm{B} 3))$ were used to cross classify the CD twins into four different categories, all of which were then compared with the healthy twin of the CD patients. Smoking was introduced into the model (three categories-not smoking, ex-smoker, and smoker) but rejected due to statistical interaction with location and behaviour. To comply with the statistical assumptions, group comparisons were carried through on the ${ }^{\mathrm{e}}$ logarithm of ASCA, and then the results were transformed back to the original ASCA scale. The effect parameter is therefore the ratio of geometric means rather than differences. ${ }^{20}$

To specifically address the question of agreement of ASCA titres within the twin pairs, we calculated the intraclass correlation coefficient (ICC) according to Dunn. ${ }^{21}$ This formulation of the ICC can be interpreted analogously to the weighted kappa index for agreement in paired measurements. A high value of ICC $(>0.80)$ indicates very good to excellent agreement whereas low ICC values $(<0.40)$ indicate poor to fair agreement. ${ }^{21}$ It is possible with this formulation of ICC, which focuses on pairwise agreement, that severe disagreement might cause ICC to be negative. Comparisons of ICC for different twin groups may thus give insight into genetic and environmental influences.

The Örebro County Ethics Committee approved the study.

\section{RESULTS \\ Twins}

A total of 151 twin pairs were invited to participate in the study. In 92 pairs $(61 \%)$ both twins in each pair agreed to take part. Additionally, six twin pairs, known by us but not belonging to the epidemiological cohorts, also agreed to take part. Thus in total 98 twin pairs participated: 58 twin pairs with CD (concordant monozygotic $\mathrm{n}=10$, discordant monozygotic $n=20$, concordant dizygotic $n=1$, and discordant 
A

$M Z$ with one $C D$ and one $U C$

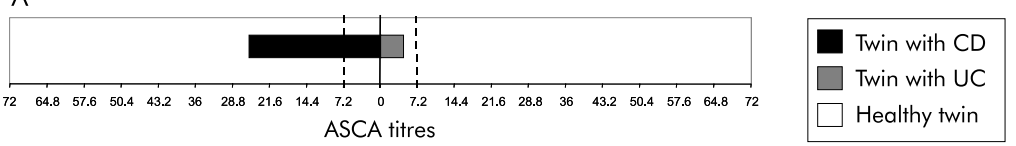

B

$M Z$ concordant $C D$

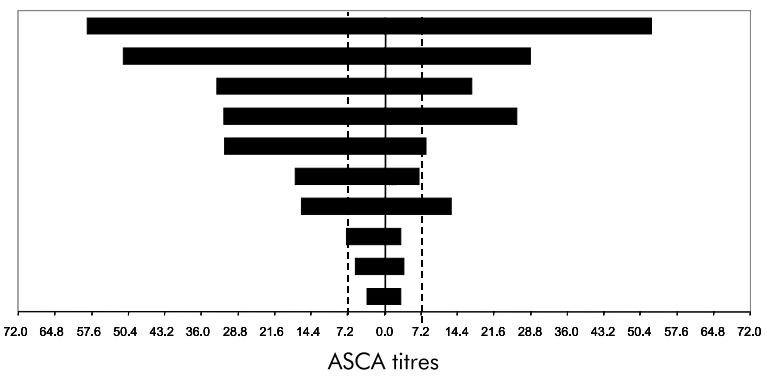

D $\quad M Z$ concordant UC

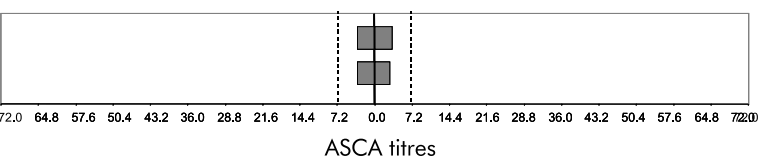

$\mathrm{E}$

MZ discordant UC

C

$M Z$ discordant $C D$

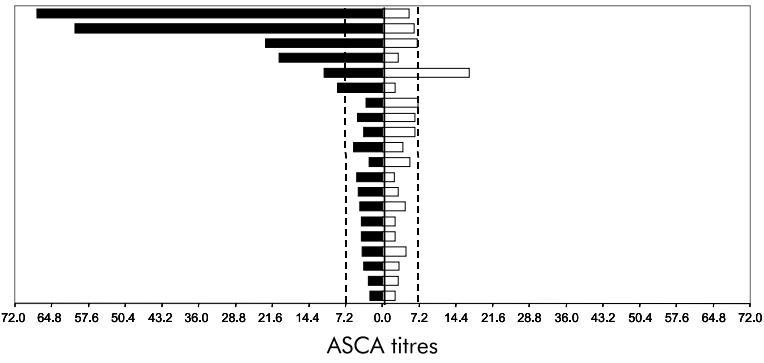

$\mathrm{F}$

DZ concordant CD
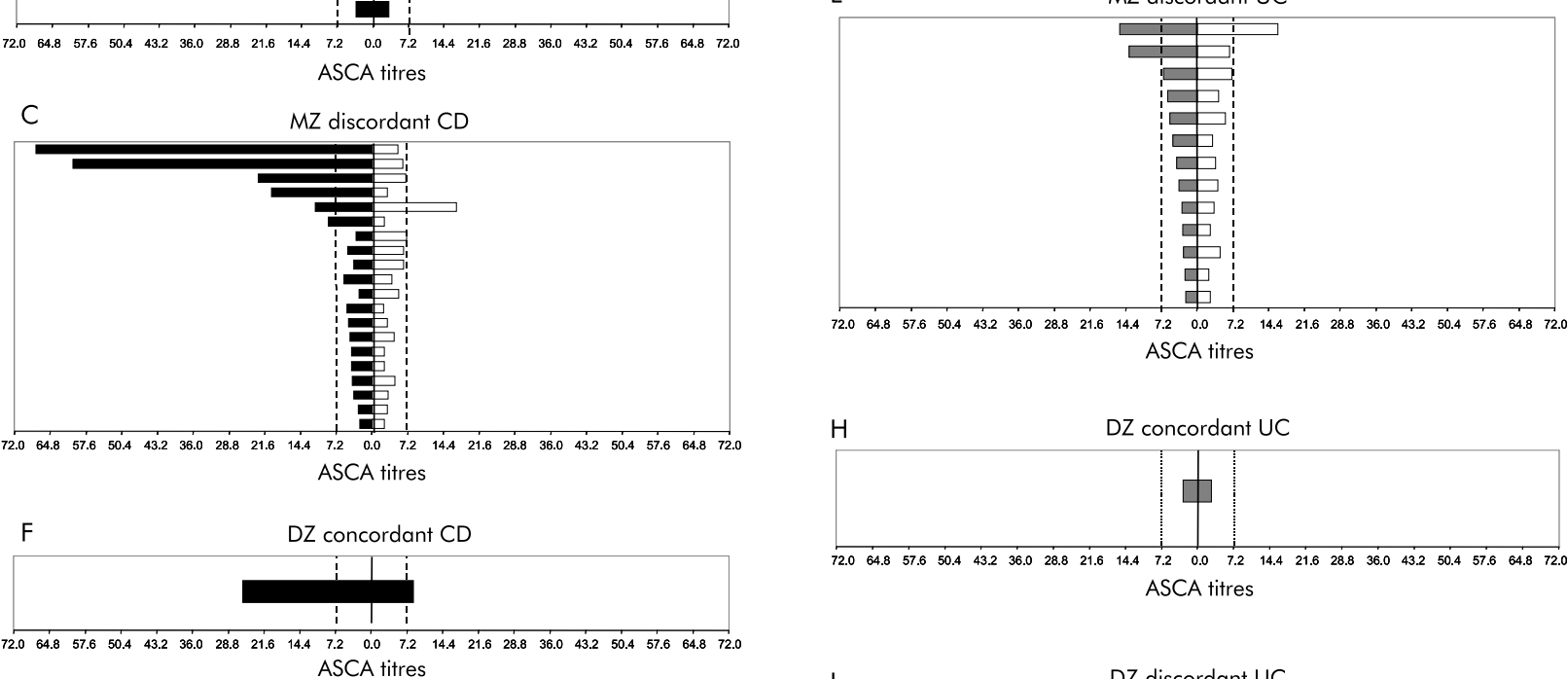

$\mathrm{H} \quad$ DZ concordant UC

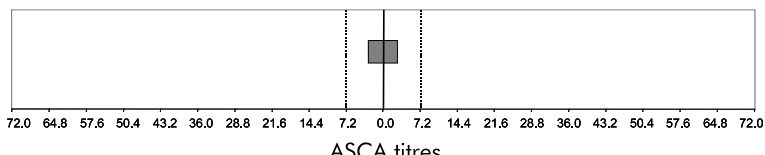

ASCA titres

G

DZ discordant CD

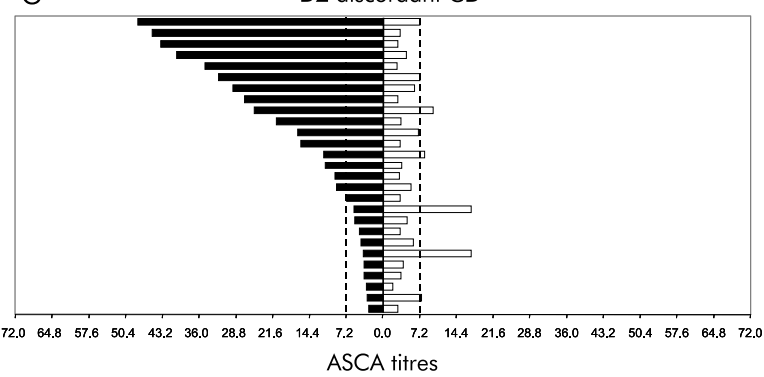

I DZ discordant UC

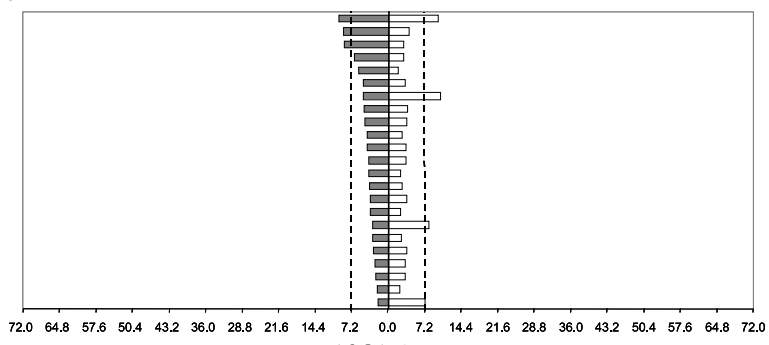

ASCA titres

Figure 1 Distribution of anti-Saccharomyces cerevisiae antibodies (ASCA) titres by pairs of twins. The broken lines correspond to the cut off values. Twin pairs are ordered along the $Y$ axis according to decreasing ASCA titres on the left panel of each graph. In twin pairs concordant for the disease, the left panel comprises the twin in each pair with the highest ASCA titre and the right panel the twin with the lower ASCA titre. In twin pairs discordant for inflammatory bowel disease, the left panel comprises the diseased twin and the right panel the healthy twin sibling. (A) Monozygotic (MZ) twin pair with one twin suffering from Crohn's disease (CD) and the other from ulcerative colitis (UC). (B) MZ twin pairs concordant for CD. (C) MZ twin pairs discordant for CD. (D) MZ twin pairs concordant for UC. (E) MZ twin pairs discordant for UC. (F) Dizygotic (DZ) twin pairs concordant for CD. (G) DZ twin pairs discordant for CD. (H) DZ twin pair concordant for UC. (I) DZ twin pairs discordant for UC.

dizygotic $\mathrm{n}=27$ ), 39 twin pairs with UC (concordant monozygotic $n=2$, discordant monozygotic $n=13$, concordant dizygotic $\mathrm{n}=\mathrm{l}$, and discordant dizygotic $\mathrm{n}=23$ ), and one monozygotic twin pair with one twin suffering from CD and one from UC. Thus a total of 70 twins had CD and 43 twins had UC. The male:female ratio was 0.75:1. Mean (range) disease duration was 21 (0-37) years since diagnosis of $\mathrm{CD}$ and $26(6-53)$ years since diagnosis of UC. A family history of IBD was reported by $8 / 70$ (11\%) twins with CD and 5/43 (12\%) twins with UC, and was not associated with the presence of ASCA (data not shown). Data on age, disease location, behaviour at diagnosis, and CARD15/NOD2 status in CD twins are presented in table 1.
ASCA status and titres in the different groups of twins ASCA were detected in 40/70 (57\%) twins with CD compared with $5 / 43(12 \%)$ twins with UC, $8 / 47$ (17\%) healthy twin siblings to twins with CD, and 5/36 (14\%) healthy twin siblings to twins with UC. There was a highly significant difference between the four groups $(p<0.0001)$. Distribution of ASCA titres in each twin pair is shown in fig l. Mean ASCA titre was 15.8 (95\% CI 13.3-18.4) U/ml in CD twins compared with 4.2 (95\% CI 0.7-7.6) U/ml in UC twins, 5.4 (95\% CI 3.7-7.0) $\mathrm{U} / \mathrm{ml}$ in healthy twin siblings to twins with $\mathrm{CD}$, and 4.3 (95\% CI 2.4-6.2) $\mathrm{U} / \mathrm{ml}$ in healthy twin siblings to twins with UC. The difference between CD and UC twins was highly significant, the ratio of their means was 2.6 (95\% CI 
Table 2 Ratio for anti-Saccharomyces cerevisiae antibodies (ASCA) mean between selected groups of twins. Estimates from mixed model with type of diagnosis as the only explanatory factor

\begin{tabular}{lllc}
\hline Groups compared & $\begin{array}{l}\text { Ratio of } \\
\text { means }\end{array}$ & $\begin{array}{l}95 \% \text { Cl for } \\
\text { ratio }\end{array}$ & $\begin{array}{l}\text { p value for testing } \\
\text { ratio }=1.0\end{array}$ \\
\hline CD-UC & 2.6 & $1.9-3.5$ & $<0.0001$ \\
CD-HT CD & 2.2 & $1.8-2.7$ & $<0.0001$ \\
UC-HT UC & 1.0 & $0.8-1.3$ & 0.73 \\
HT CD-HT UC & 1.2 & $1.0-1.6$ & 0.10 \\
CD colonic (L2)-CD small bowel (L1/3/4) & 0.5 & $0.3-0.7$ & $<0.0001$ \\
CD colonic (L2)-HT CD colonic & 1.2 & $0.9-1.7$ & 0.29 \\
CD small bowel (L1/3/4)-HT CD small bowel & 3.0 & $2.3-3.8$ & $<0.0001$ \\
HT CD colonic-HT CD small bowel & 1.1 & $0.8-1.6$ & 0.47 \\
CD non-strict non-penetr (B1)-CD strict/penetr (B2/3) & 0.5 & $0.4-0.7$ & 0.0004 \\
CD non-strict non-penetr (B1)-HT CD non-strict non-penetr & 1.4 & $1.0-1.9$ & 0.03 \\
CD strict/penetr (B2/3)-HT CD strict/penetr & 3.1 & $2.4-4.0$ & $<0.0001$ \\
CD $<40$ y (A1)-CD $\geq 40$ y (A2) & 2.1 & $1.4-3.2$ & 0.0005 \\
CD <40 y (A1)-HT CD $<40$ y & 2.5 & $2.0-3.1$ & $<0.0001$ \\
CD $\geqslant 40$ y (A2)-HT CD $\geq 40$ y & 1.5 & $1.0-2.4$ & 0.05 \\
CD CARD15 mutated-CD CARD15 wild-type & 0.66 & $0.4-1.1$ & 0.09 \\
CD CARD15 mutated-HT CD CARD15 mutated & 1.67 & $1.0-2.9$ & 0.07 \\
CD CARD15 wild-type-HT CD CARD15 wild-type & 2.37 & $1.9-3.0$ & $<0.0001$ \\
\hline
\end{tabular}

$C D$, Crohn's disease; UC, ulcerative colitis; HT CD, healthy twin sibling to twin with Crohn's disease; HT UC, healthy twin sibling to twin with ulcerative colitis; non-strict non-penetr, non-stricturing non-penetrating; strict/ penetr, stricturing or penetrating; $95 \% \mathrm{Cl}, 95 \%$ confidence interval.

1.9-3.5; $p>0.0001$ ), and the difference between CD twins and their healthy twin siblings was also statistically significant (ratio 2.2; 95\% CI 1.8-2.7; $\mathrm{p}<0.0001$ ). UC twins and healthy twin siblings showed no difference (table 2). There was no increased occurrence of ASCA in healthy twin siblings in discordant monozygotic twin pairs with CD compared with dizygotic ones. Contrary to expectations, ASCA were found in $1 / 20(5 \%)$ discordant monozygotic pairs and in 7/27 (26\%) discordant dizygotic pairs $(\mathrm{p}=0.11)$. However, no independent association was found between ASCA titres and zygosity in the multivariate analysis.

\section{ASCA status and titres according to CD phenotypes Location}

Distribution of ASCA titres according to disease location is given in fig 2. ASCA were present in 6/21 (29\%) twins with pure colonic CD (L2) and in 34/49 (69\%) twins with small bowel involvement $(\mathrm{L} 1+\mathrm{L} 3+\mathrm{L} 4)(\mathrm{p}=0.003)$. Similarly, the mean ASCA titre was 10.1 (95\% CI 5.4-14.8) U/ml in twins with pure colonic CD (L2) and 18.2 (95\% CI 15.2-21.1) U/ml in CD twins with small bowel involvement $(\mathrm{Ll}+\mathrm{L} 3+\mathrm{L} 4)$ with a highly significant difference in the ratio of means (ratio 0.5; 95\% CI 0.3-0.7; p<0.0001) (table 2). Furthermore, ASCA titres in twins with small bowel involvement were higher than in their healthy twin siblings (ratio 3.0; 95\% CI 2.3-3.8; $\mathrm{p}<0.0001$ ) (table 2). However, there was no significant difference between twins with pure colonic $C D$ and their healthy twin siblings $(p=0.29)$ (table 2 ). There was no difference between healthy twin siblings of twins with pure colonic CD and healthy twin siblings of twins with small bowel involvement in ASCA status $(2 / 17 v 6 / 30 ; \mathrm{p}=0.69)$ or in ASCA titres ( $6.3 \vee 4.7 \mathrm{U} / \mathrm{ml} ; \mathrm{p}=0.47)$.

\section{Behaviour}

Distribution of ASCA titres according to disease behaviour is given in fig 2. ASCA were present in 12/30 (40\%) twins with non-stricturing non-penetrating CD (B1) and in 28/40 (70\%) twins with complicated, either stricturing (B2) or penetrating (B3), CD $(p=0.016)$. Similarly, mean ASCA titres were lower in twins with non-stricturing non-penetrating CD (B1) (11.4 (95\% CI 7.5-15.4) U/ml) than in twins with complicated disease (B2 or B3) ( 18.8 (95\% CI 15.6-22.0) U/ml) with a ratio of 0.5 (95\% CI 0.4-0.7; p=0.0004). For comparisons of twins with CD and their healthy twin siblings, see table 2 .
Age

Distribution of ASCA titres according to age at diagnosis is given in fig 2. ASCA were present in 35/55 (64\%) twins with age at diagnosis $<40$ years ( $\mathrm{Al}$ ) and in $5 / 15(33 \%)$ twins with age at diagnosis $\geqslant 40$ years $(\mathrm{A} 2)(\mathrm{p}=0.04)$. Similarly, the mean ASCA titre was 18.9 (95\% CI 16.1-21.8) U/ml in twins with age at diagnosis <40 years (Al) and 8.0 (95\% CI 3.5$12-5) \mathrm{U} / \mathrm{ml}$ in twins with age at diagnosis $\geqslant 40$ years (A2) (ratio 2.1; 95\% CI 1.4-3.2; p=0.0005) (table 2). Analysing ASCA in even younger CD twins ( $<30$ years of age at diagnosis) did not change the results; ASCA were present in $27 / 43(63 \%)$ and mean titre was 18.8 (95\% CI 15.6-22.0) $\mathrm{U} / \mathrm{ml}$. For comparisons of twins with $\mathrm{CD}$ and their healthy twin siblings, see table 2.

\section{CARD15/NOD2}

CARD15/NOD2 status was available in 68 of $70 \mathrm{CD}$ twins. ASCA were equally distributed in CD twins with any of the three single nucleotide polymorphisms, Arg702Trp, Gly908Arg, or Leu1007fsinsC, as in CD twins with the wildtype genotype $(6 / 12(50 \%) \vee 33 / 56(59 \%) ; p=0.75)$ (fig 2 ). Mean ASCA titre was lower however, although not statistically significant, in twins carrying any of these polymorphisms (table 2).

\section{Multivariate perspective of phenotype ASCA association}

As clinical characteristics in CD are dependent on each other, analyses with a quantitative multivariate perspective were added. In twins with pure colonic disease (L2) and a nonstricturing non-penetrating behaviour (B1), ASCA titres were similar to those in healthy twin siblings (table 3). In contrast, twins with either complicated CD behaviour (B2 or B3) or small bowel involvement (L1, L3, or L4) had significantly higher ASCA titres than their healthy twin siblings $(p=0.008$ and $\mathrm{p}=0.004$, respectively).

\section{Agreement on ASCA titres within twin pairs}

ASCA titres matched for each twin pair depending on zygosity, concordance, or discordance for IBD status are shown in fig 1 . To assess the agreement of ASCA titres within twin pairs, the ICC was used. Greater agreement within monozygotic pairs with IBD ( ICC $=0.44)$ than dizygotic pairs $(\mathrm{ICC}=-0.06)$ was observed (table 4$)$. Within concordant 

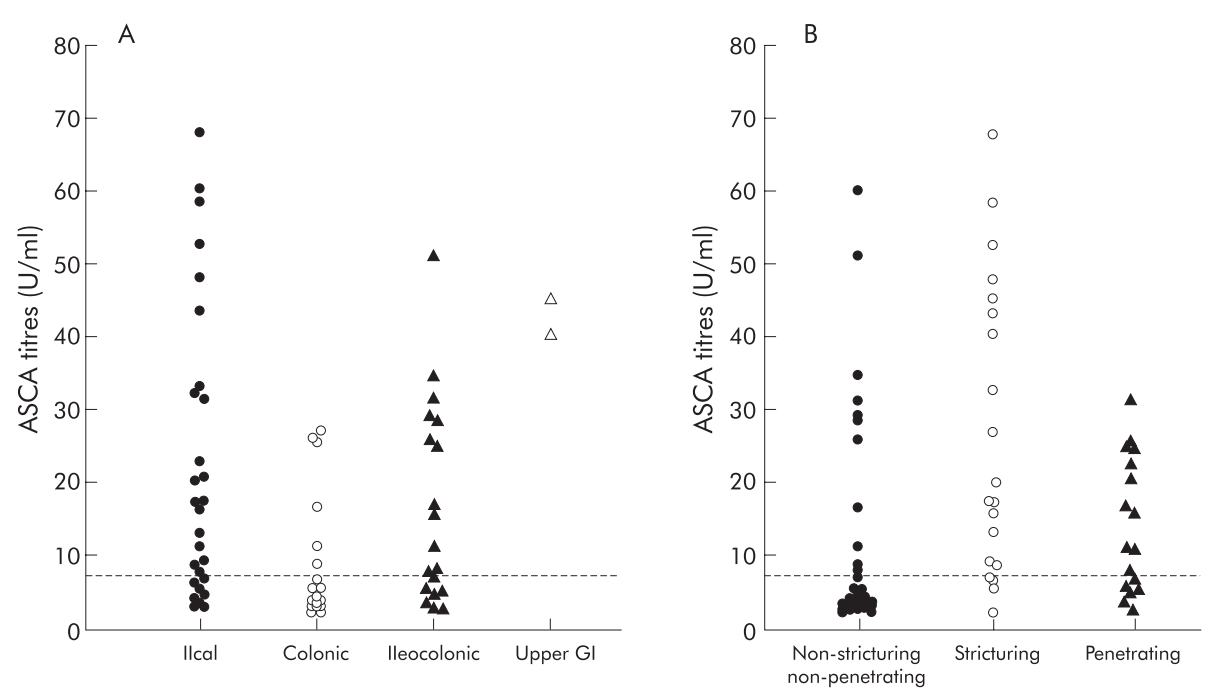

Figure 2 Distribution of antiSaccharomyces cerevisiae antibodies (ASCA) according to location (A), behaviour (B), age at diagnosis of Crohn's disease (C), and CARD15/ NOD2 genotype (D)
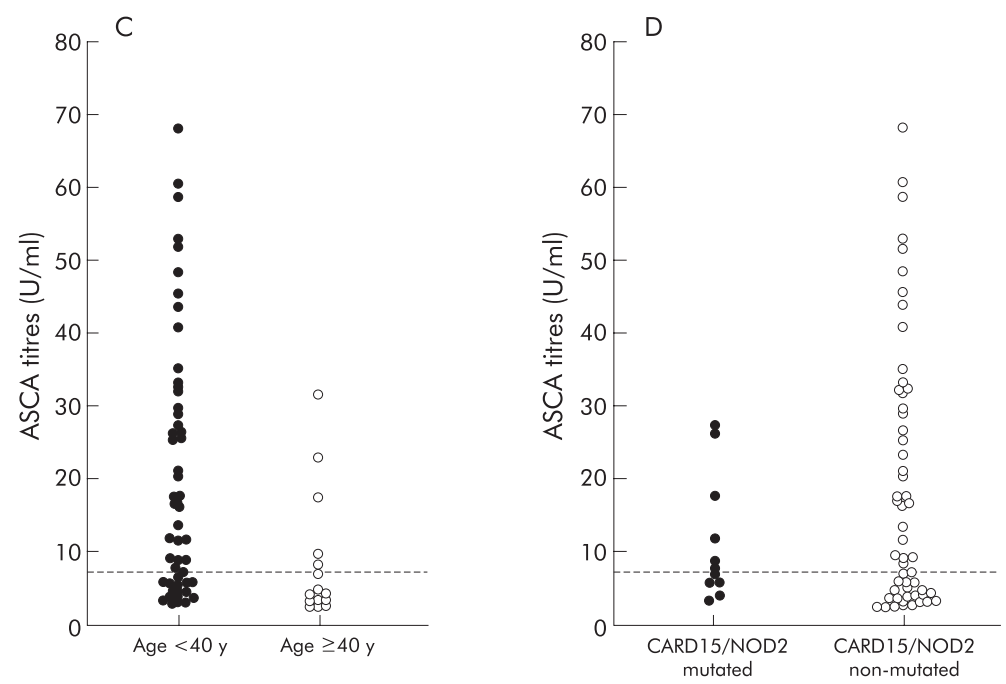

monozygotic twin pairs with $\mathrm{CD}$, a high value was observed $($ ICC $=0.76)$. As there was only one concordant dizygotic pair with CD, ICC could not be calculated in this subgroup of twins. In both monozygotic and dizygotic twin pairs discordant for CD, low ICC values were observed (ICC $=-0.02$ and ICC $=-0.26$, respectively). Furthermore, in UC twin pairs, ICC was very high in discordant monozygotic but not in dizygotic pairs with UC (ICC $=0.84$ and ICC $=0.21$, respectively).

\section{DISCUSSION}

Based on family studies showing an increased occurrence of ASCA in healthy relatives, it has been proposed that ASCA may be a susceptibility marker for CD. Monozygotic twin pairs are genetically identical, in contrast with dizygotic twin pairs and siblings. However, in the present study, ASCA were found in only $1 / 20$ healthy twin siblings in discordant monozygotic twin pairs with CD compared with 7/27 healthy twin siblings in discordant dizygotic pairs. Furthermore, no

Table 3 Analysis of anti-Saccharomyces cerevisiae antibodies (ASCA) in twins classified with Crohn's disease (CD) in multivariate categories (stratification for location and behaviour)

\begin{tabular}{lcll}
\hline Category & n & $\begin{array}{l}\text { Adjusted mean } \\
(95 \% \text { Cl) of } \\
\text { ASCA (U/ml) }\end{array}$ & $\begin{array}{l}\text { Ratio }(95 \% \mathrm{Cl} \text { of } \mathrm{ASCA} \\
\text { for CD } \mathbf{v} \text { healthy twin } \\
\text { sibling; } \mathrm{p} \text { value }\end{array}$ \\
\hline CD, colonic (L2), non-strict non-penetr (B1) & 14 & $4.8(-2.6-12.2)$ & $0.9(0.6-1.5) ; p=0.73$ \\
CD, colonic (L2), strict/penetr (B2/3) & 7 & $17.1(8.1-26.2)$ & $2.2(1.2-4.1) ; p=0.008$ \\
CD, small bowel (L1, 3, 4), non-strict non-penetr (B1) & 16 & $16.0(9.8-22.1)$ & $2.2(1.4-3.4) ; p=0.004$ \\
CD, small Bowel (L1, 3, 4), strict/penetr (B2/3) & 33 & $19.3(15.0-23.6)$ & $3.2(2.4-4.4) ; p<0.0001$ \\
HT CD & 47 & $5.2(3.2-7.2)$ & 1.0 (reference) \\
\hline
\end{tabular}

$C D, C r o h n ' s$ disease; non-strict non-penetr, non-stricturing non-penetrating; strict/penetr, stricturing or penetrating; HT CD, healthy twin sibling to twin with Crohn's disease.

Adjusted mean values of ASCA and ratios of ASCA mean between CD categories and healthy twins (reference category).

$95 \% \mathrm{Cl}, 95 \%$ confidence intervals as well as parameter estimates. 
Table 4 Intraclass correlation coefficient of antiSaccharomyces cerevisiae antibodies in different twin groups

\begin{tabular}{llr}
\hline & No of twin pairs & \multicolumn{1}{c}{ ICC } \\
\hline IBD MZ & 46 & 0.44 \\
IBD DZ & 52 & -0.06 \\
CD MZ concordant & 10 & 0.76 \\
CD MZ discordant & 20 & -0.02 \\
CD DZ discordant & 27 & -0.26 \\
UC MZ discordant & 13 & 0.84 \\
UC DZ discordant & 23 & 0.21 \\
\hline
\end{tabular}

IBD $M Z$, monozygotic twin pairs with inflammatory bowel disease; IBD $D Z$, dizygotic twin pairs with inflammatory bowel disease; $C D M Z$ concordant, concordant monozygotic twin pairs with Crohn's disease; CD MZ discordant, discordant monozygotic twin pairs with Crohn's disease; CD DZ discordant, discordant dizygotic twin pairs with Crohn's disease; UC MZ discordant, discordant monozygotic twin pairs with ulcerative colitis; UC DZ discordant, discordant dizygotic twin pairs with ulcerative colitis.

similarity in ASCA titres was observed within discordant monozygotic twin pairs with CD ( ICC $=-0.02$ ) or discordant dizygotic pairs (ICC $=-0.26$ ) and no independent association was found between ASCA titres and zygosity in the multivariate analysis. These findings question the concept of ASCA as a marker of genetic susceptibility for CD and rather suggest that ASCA in healthy family members is a marker of shared environment.

Overall, ASCA were found in $57 \%$ of CD twins, in $17 \%$ of healthy twin siblings to twins with $\mathrm{CD}$, and in $12 \%$ of UC twins, which is consistent with previous findings. ${ }^{16-9} 22-24$ Consistently, qualitative phenotypic associations with small bowel disease, ${ }^{13}$ complicated (either stricturing or penetrating) $\mathrm{CD}_{1}{ }^{23}$ and young age at diagnosis ${ }^{12}$ were also apparent in the twin cohort. Quantitative associations between ASCA titres and young age at diagnosis, small bowel involvement, and complicated disease behaviour ${ }^{2}{ }^{4}$ were also confirmed in the univariate analyses in the twin cohort. However, the limited number of twins in each subgroup did not allow us to evaluate the relative contribution of all three clinical characteristics to ASCA titre in a multivariate perspective. It has recently been proposed that complicated disease behaviour is associated with an increased serological response. ${ }^{4}$ Therefore, disease behaviour and location were included in the multivariate analysis. Interestingly, no difference in ASCA titres was observed between CD twins with nonstricturing non-penetrating (B1) pure colonic disease (L2) and their healthy twin siblings (table 3 ). In contrast, twins with either complicated CD behaviour (B2 or B3) or small bowel involvement (L1, L3, or L4) had significantly higher ASCA titres than their healthy twin siblings. Consequently, the highest ASCA levels were found in twins with small bowel involvement and complicated disease behaviour.

The ICC for pairwise observations was used to assess agreement in ASCA titres within twin pairs. In the overall analysis, a moderate correlation was found in monozygotic twin pairs $(\mathrm{ICC}=0.44)$ but not in dizygotic pairs $($ ICC $=-0.06)$. This suggests that levels of ASCA are similar within monozygotic but not within dizygotic twin pairs. Furthermore, there was strong agreement in ASCA titres within the concordant $\mathrm{CD}$ pairs (ICC $=0.76$ ). In contrast, ASCA titres were $26.0 \mathrm{U} / \mathrm{ml}$ and $8.4 \mathrm{U} / \mathrm{ml}$, respectively, in the concordant dizygotic pair with CD. As only one pair took part in the study, ICC could not be calculated. These findings and the absence of ASCA in healthy twin siblings in discordant monozygotic pairs with CD suggest that CD is associated with an increase in ASCA titres and that the level of increase seems to be genetically determined.
No differences were found in either ASCA status $(\mathrm{p}>0.99)$ or ASCA titres $(p=0.99)$ between UC twins and their healthy twin siblings. Interestingly, a high correlation for ASCA titres was also observed in discordant monozygotic UC pairs (ICC $=0.84$ ) but not in discordant dizygotic twins $($ ICC $=0.21)$.

It has recently been hypothesised that relevant commensal bacteria can trigger and perpetuate a more complicated disease behaviour in genetically susceptible subjects. ${ }^{4}$ Furthermore, it has been proposed that immune responses are closer to the pathophysiological pathway of complicated disease behaviour than genetic susceptibility. However, concordance in ASCA titres in concordant monozygotic twin pairs with $C D$ also suggests that genes determine the level of ASCA response.

The CARD15/NOD2 gene has been identified as an important determinant of susceptibility to CD. ${ }^{25}{ }^{26}$ There are marked geographic differences in the occurrence of CARD15/ NOD2 polymorphisms. ${ }^{27}{ }^{28}$ The low frequency observed in the twin cohort is in accordance with data from other Scandinavian ${ }^{29}{ }^{30}$ and Northern European countries, ${ }^{28}$ in contrast with other parts of Europe 25 $2931-33^{3}$ and North America. ${ }^{34-38}$ CARD15/NOD2 polymorphisms are associated with the same CD phenotype as ASCA—namely, ileal disease, young age at diagnosis, and possibly also stricturing CD. ${ }^{29} 3133-353940$ It has also been argued that CARD15/NOD2 polymorphisms are independently associated with the presence of ASCA. ${ }^{41}{ }^{42}$ This is however controversial and has not been replicated by others. ${ }^{35}$ We were not able to find any qualitative or quantitative association in the twin cohort, which suggests that other genes determine ASCA titres. Interesting data on the influence of the genetics of ASCA were recently reported. ${ }^{43}$ In a subgroup of $\mathrm{CD}$ patients carrying mutations in the exon of the mannan binding lectin gene, production of mannan binding lectin was low and those patients were more often ASCA positive.

In summary, no increased occurrence of ASCA was observed in healthy twin siblings in discordant monozygotic twin pairs. This questions the concept of ASCA as a marker of genetic susceptibility for CD and rather points towards ASCA as a marker of shared environment. A high degree of concordance in ASCA titres was observed in concordant monozygotic twin pairs with $C D$, suggesting that the level of increase is genetically determined. Based on these findings we propose that ASCA are a marker of a response to an environmental antigen and that a specific gene(s) other than CARD15/NOD2 determines the level of response and perhaps also specific phenotypic characteristics.

\section{ACKNOWLEDGEMENTS}

This work was supported by a grant from Bengt Ihre's Foundation, Örebro University Hospital Research Foundation, and Örebro County Research Foundation.

\section{Authors' affiliations}

J Halfvarson, G Järnerot, Division of Gastroenterology, Department of Internal Medicine, Örebro University Hospital, Örebro, Sweden A Standaert-Vitse, B Sendid, T Jouault, D Poulain, Laboratoire de Mycologie Fondamentale and Appliquée, Inserm E360, Faculté de Médecine, CHU Lille, Lille, France

L Bodin, Statistical and Epidemiological Unit, Clinical Research Centre, Örebro University Hospital, Örebro, Sweden

A Duhamel, Centre d'Etudes et de Recherche en Informatique Médicale, Faculté de Médecine, CHU Lille, Lille, France

J F Colombel, Department of Hepatogastroenterology, Hopital Huriez, CHU Lille, Lille, France

C Tysk, Division of Gastroenterology, Department of Internal Medicine, and Department of Clinical Medicine, Örebro University Hospital, Örebro, Sweden

Conflict of interest: None declared. 


\section{REFERENCES}

1 Quinton JF, Sendid B, Reumaux D, et al. Anti-Saccharomyces cerevisiae mannan antibodies combined with antineutrophil cytoplasmic autoantibodies in inflammatory bowel disease: prevalence and diagnostic role. Gut 1998;42:788-91

2 Vasiliauskas EA, Kam LY, Karp LC, et al. Marker antibody expression stratifie Crohn's disease into immunologically homogeneous subgroups with distinct clinical characteristics. Gut 2000;47:487-96.

3 Walker L, Aldhous MC, Drummond HE, et al. Anti-Saccharomyces cerevisiae antibodies (ASCA) in Crohn's disease are associated with disease severity but not NOD2/CARD1 5 mutations. Clin Exp Immunol 2004;135:490-6.

4 Mow WS, Vasiliauskas EA, Lin YC, et al. Association of antibody responses to microbial antigens and complications of small bowel Crohn's disease. Gastroenterology 2004;126:414-24.

5 Russell RK, Satsangi J. IBD: a family affair. Best Pract Res Clin Gastroenterol 2004; 18:525-39.

6 Sendid B, Quinton JF, Charrier G, et al. Anti-Saccharomyces cerevisiae mannan antibodies in familial Crohn's disease. Am J Gastroenterol 1998;93:1306-10.

7 Seibold F, Stich O, Hufnagl R, et al. Anti-Saccharomyces cerevisiae antibodies in inflammatory bowel disease: a family study. Scand J Gastroenterol 2001;36:196-201

8 Sutton CL, Yang H, Li Z, et al. Familial expression of anti-Saccharomyces cerevisiae mannan antibodies in affected and unaffected relatives of patients with Crohn's disease. Gut 2000:46:58-63.

9 Vermeire S, Peeters M, Vlietinck R, et al. Anti-Saccharomyces cerevisiae antibodies (ASCA), phenotypes of IBD, and intestinal permeability: a study in IBD families. Inflamm Bowel Dis 2001;7:8-15.

10 Tysk $C$, Lindberg $E$, Järnerot $G$, et al. Ulcerative colitis and Crohn's disease in an unselected population of monozygotic and dizygotic twins. A study of heritability and the influence of smoking. Gut 1988;29:990-6.

11 Halfvarson J, Bodin L, Tysk C, et al. Inflammatory bowel disease in a Swedish twin cohort: a long-term follow-up of concordance and clinical characteristics. Gastroenterology 2003:124:1767-73.

12 Halfvarson J, Tysk C, Järnerot G. Decreasing pair concordance in monozygotic twins with Crohns disease. Gastroenterology 2004; 126(suppl 2):A45

13 Gasche C, Scholmerich J, Brynskov J, et al. A simple classification of Crohn's disease: report of the Working Party for the World Congresses of Gastroenterology, Vienna 1998. Inflamm Bowel Dis 2000;6:8-15.

14 Cederlöf R, Friberg L, Jonsson E, et al. Studies on similarity diagnosis in twins with the aid of mailed questionnaires. Acta Genet 1961;11:338-62.

15 Lichtenstein P, De Faire U, Floderus B, et al. The Swedish Twin Registry: a unique resource for clinical, epidemiological and genetic studies. J Intern Med 2002;252:184-205

16 Sendid B, Colombel JF, Jacquinot PM, et al. Specific antibody response to oligomannosidic epitopes in Crohn's disease. Clin Diagn Lab Immunol 1996:3:219-26.

17 Halfvarson J, Bresso F, Tysk C, et al. Genetic Crohn's disease. A different entity than sporadic. A study in monozygotic twins. Gastroenterology 2004; 126(suppl 2):A359.

18 Halfvarson J, Daperno M, Ahmad T, et al. Influenc of CARD15/NOD2 and IBD5 in Swedish dizygotic twins (Swedish). Sv Läkarsällskapets Handl Hygiea 2003; 112:168

19 Brown H, Prescott R. Applied mixed models in medicine. Chichester, UK: John Wiley and Sons, 1999:199-235.

20 Bland JM, Altman DG. Statistics notes: The use of transformation when comparing two means. BMJ 1996;312:1153.

21 Dunn G. Design and analysis of reliability studies. The statistical evaluation of measurement errors. New York: Oxford University Press, 1989:34-5.

22 Main J, McKenzie H, Yeaman GR, et al. Antibody to Saccharomyces cerevisiae (bakers' yeast) in Crohn's disease. BMJ 1988;297:1105-6.
23 Ruemmele FM, Targan SR, Levy G, et al. Diagnostic accuracy of serological assays in pediatric inflammatory bowel disease. Gastroenterology 1998;115:822-9.

24 Peeters $M$, Joossens S, Vermeire S, et al. Diagnostic value of antiSaccharomyces cerevisiae and antineutrophil cytoplasmic autoantibodies in inflammatory bowel disease. Am J Gastroenterol 2001;96:730-4.

25 Hugot JP, Chamaillard M, Zouali $\mathrm{H}$, et al. Association of NOD2 leucine-rich repeat variants with susceptibility to Crohn's disease. Nature 2001;411:599-603.

26 Ogura $\mathrm{Y}$, Bonen DK, Inohara N, et al. A frameshift mutation in NOD2 associated with susceptibility to Crohn's disease. Nature 2001;411:603-6.

27 Ahmad T, Tamboli CP, Jewell D, et al. Clinical relevance of advances in genetics and pharmacogenetics of IBD. Gastroenterology 2004:126:1533-49.

28 Arnott ID, Nimmo ER, Drummond HE, et al. NOD2/CARD15, TLR4 and CD14 mutations in Scottish and Irish Crohn's disease patients: evidence for genetic heterogeneity within Europe? Genes Immun 2004;5:417-25

29 Hampe J, Grebe J, Nikolaus S, et al. Association of NOD2 (CARD 15) genotype with clinical course of Crohn's disease: a cohort study. Lancet 2002:359:1661-5

30 Helio T, Halme L, Lappalainen M, et al. CARD15/NOD2 gene variants are associated with familially occurring and complicated forms of Crohn's disease. Gut 2003;52:558-62

31 Ahmad T, Armuzzi A, Bunce $M$, et al. The molecular classification of the clinical manifestations of Crohn's disease. Gastroenterology 2002;122:854-66

32 Vermeire S, Louis E, Rutgeerts $\mathrm{P}$, et al. NOD2/CARD15 does not influence response to infliximab in Crohn's disease. Gastroenterology 2002;123:106-11.

33 Cuthbert AP, Fisher SA, Mirza MM, et al. The contribution of NOD2 gene mutations to the risk and site of disease in inflammatory bowel disease. Gastroenterology 2002;122:867-74

34 Vermeire S, Wild G, Kocher K, et al. CARD15 genetic variation in a Quebec population: prevalence, genotype-phenotype relationship, and haplotype structure. Am J Hum Genet 2002;71:74-83.

35 Abreu MT, Taylor KD, Lin YC, et al. Mutations in NOD2 are associated with fibrostenosing disease in patients with Crohn's disease. Gastroenterology 2002; 123:679-88

36 Zhou Z, Lin XY, Akolkar PN, et al. Variation at NOD2/CARD15 in familial and sporadic cases of Crohn's disease in the Ashkenazi Jewish population. Am J Gastroenterol 2002;97:3095-101.

37 Sugimura K, Taylor KD, Lin YC, et al. A novel NOD2/CARD15 haplotype conferring risk for Crohn disease in Ashkenazi Jews. Am J Hum Genet 2003;72:509-18.

38 Bonen DK, Ogura Y, Nicolae DL, et al. Crohn's disease-associated NOD2 variants share a signaling defect in response to lipopolysaccharide and peptidoglycan. Gastroenterology 2003;124:140-6.

39 Lesage S, Zouali H, Cezard JP, et al. CARD15/NOD2 mutational analysis and genotype-phenotype correlation in 612 patients with inflammatory bowel disease. Am J Hum Genet 2002;70:845-57.

40 Radlmayr M, Torok HP, Martin K, et al. The c-insertion mutation of the NOD2 gene is associated with fistulizing and fibrostenotic phenotypes in Crohn's disease. Gastroenterology 2002;122:2091-2.

41 Dubinsky M, Lee-Uy N, Lin Y-C, et al. Synergism of NOD2/CARD 15 and ASCA (anti-Saccharomyces cerevisiae antibodies) contributes to disease behavior in pediatric Crohn's disease (CD) patients. Gastroenterology 2003;124(suppl 1):A372.

42 Esters N, Pierik M, van Steen K, et al. Transmission of CARD15 (NOD2) variants within families of patients with inflammatory bowel disease. Am J Gastroenterol 2004;99:299-305.

43 Seibold F, Konrad A, Flogerzi B, et al. Genetic variants of the mannanbinding lectin are associated with immune reactivity to mannans in Crohn's disease. Gastroenterology 2004; 127:1076-84.

\section{Call for papers}

11 th European Forum on Quality Improvement in Health Care 26-28 April 2006, Prague, Czech Republic Deadline 30 September 2005.

For further information and to submit online go to: www.quality.bmipg.com 